\title{
The architectural divergence problem in security and privacy of eHealth loT product lines
}

\author{
Oleksandr Tomashchuk \\ oleksandr.tomashchuk@philips.com \\ Philips Research \\ Eindhoven, Netherlands \\ imec-DistriNet, KU Leuven \\ Heverlee, Belgium
}

\author{
Dimitri Van Landuyt \\ dimitri.vanlanduyt@kuleuven.be \\ imec-DistriNet, KU Leuven \\ Heverlee, Belgium
}

\author{
Wouter Joosen \\ wouter.joosen@kuleuven.be \\ imec-DistriNet, KU Leuven \\ Heverlee, Belgium
}

\begin{abstract}
The Internet of Things (IoT) seamlessly becomes integrated into many aspects of daily life, and in the case of healthcare, it arises in the shape of eHealth IoT systems. Evidently, the design of such systems must apply best practices when it comes to security and privacy, in addition to ensuring compliance with various national and international regulations. From the perspective of the required functionality, commonalities and variations can effectively be managed by adopting a product line approach that involves deriving specific application architecture variants from a common reference architecture.

This paper illustrates and discusses a specific problem encountered in the establishment of a software product-line in this specific context: the adoption of systematic security and privacy threat modeling and risk assessment approaches introduces a variation space that is very difficult to capture in a proactive product-line approach. One of the main causes for this is that threat assessment itself suffers from the problem of threat explosion, i.e. combinatorial explosions of threats that have to be investigated and systematically mitigated. The highlighted divergence of the security and privacy threats across architectural variants is illustrated in the specific case of an industry IoT-based e-health software product line.
\end{abstract}

\section{CCS CONCEPTS}

- Security and privacy $\rightarrow$ Information flow control; Economics of security and privacy; $\bullet$ Software and its engineering $\rightarrow$ Searchbased software engineering; Risk management; Software product lines.

\section{ACM Reference Format:}

Oleksandr Tomashchuk, Dimitri Van Landuyt, and Wouter Joosen. 2021. The architectural divergence problem in security and privacy of eHealth IoT product lines. In SPLC '21: Proceedings of the 24th ACM International Systems and Software Product Line Conference. ACM, New York, NY, USA, 6 pages. https://doi.org/00.0000/0000000.0000000

Permission to make digital or hard copies of all or part of this work for personal or classroom use is granted without fee provided that copies are not made or distributed for profit or commercial advantage and that copies bear this notice and the full citation on the first page. Copyrights for components of this work owned by others than ACM must be honored. Abstracting with credit is permitted. To copy otherwise, or republish, to post on servers or to redistribute to lists, requires prior specific permission and/or a fee. Request permissions from permissions@acm.org.

SPLC 2021, September 06-11, 2021, Leicester, United Kingdom

(c) 2021 Association for Computing Machinery.

ACM ISBN 000-0-0000-XXXX-X/00/00 . \$ \$00.00

https://doi.org/00.0000/0000000.0000000

\section{INTRODUCTION}

The digitalization of modern society drastically increases the complexity and impact of current IT systems. Especially in the context of the Internet-of-Things (IoT), systems rely on a large amount and a wide variety of computational devices. Coping with such complexity often drives industrial players towards adoption of software product lines. Organizations that successfully employed SPLs, significantly benefit from improved efiiciency in terms of quality, customizability, and development costs for complex products. However, software product lines are not a silver bullet, and new challenges are being imposed.

An IoT-based ecosystem is open and dynamic, in the sense that devices come and go, personal data is collected systematically and processed and disclosed to a number of organizations for a wide variety of reasons. Such a context exacerbates the importance of keeping an end-to-end perspective on potential security and privacy threats, as well as maintaining actualized views on the inherent risk of these operations, from the complementary perspectives of security, as well as privacy. Nonetheless, the consequences to data privacy when personal data is leaked, as well as the potential security implications, for example by weaponizing IoT environments are not be underestimated, and a true security- and/or privacy-bydesign is required, identifying design flaws that may exacerbate these problems before such systems are put into practice and systematically addressing them.

Threat modelling is a process that takes place in the early stages of the development life cycle, between the analysis of key requirements and the establishment of a secure and privacy preserving software architecture. In the essence, it yields a set of identified threats, together with their estimated priority -commonly a combination of impact and likelihood but often also based on more specialized risk assessment schemes. Explicitly addressing these threats as an integral part of the development life-cycle then ensures that appropriate mitigations, countermeasures and controls are put in place to minimize the operational risk.

However, threat modelling and risk assessment are costly analysis activities, and the reuse potential of efforts spent on an individual system is limited, due to the fact that threat scenarios and risk estimation take into account many elements that are heavily application-specific, such as a valuation of involved or affected assets, existing mitigations, trust boundaries and trust assumptions.

In consequence, even in systems that are heavily based on a standardized reference architecture, small architectural variations lead to significant differences in the security and/or privacy threat landscape when compared between variants. In this paper, we highlight 


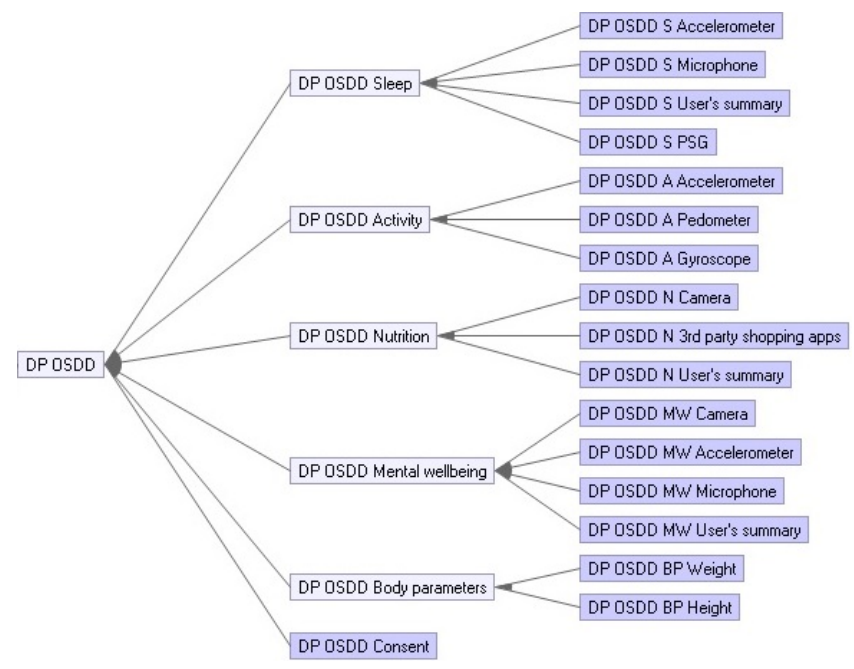

Figure 1: Part of the feature model that represents data processing within the OS-driven device

and concretize this problem, which we refer to as the 'architectural divergence problem' in security and privacy of eHealth IoT architectures.

The context for our research efforts is set by the HEART project [1], which involves the development of an eHealth IoT platform that relies on various devices and data sources. The goal of the platform is to accommodate multiple architectural variants in support of a wide array of eHealth use cases, ranging from providing analytics on multiple types of sensor data for providing coaching advices to providing governmental institutions or other recognized stakeholders with health-related statistics on a certain population.

The creation of the above mentioned platform and its architectural variants is combined with efforts to implement best practices of security and privacy by design. Therefore, with this regard we use an approach which is based on STRIDE [11], LINDDUN [6] as well as other techniques to ensure satisfaction of related requirements and compliance. As we perform threat analysis for a wide range of architectural variants that stem from the same product line, we employ a threat modelling tool called SPARTA [18] which automates both threat elicitation and risk-based assessment.

The remainder of the paper is structured as follows: Section 2 provides insights on the HEART platform and selected approach to security and privacy assessments. Section 3 introduces and demonstrates the problem that we faced, and Section 4 highlights previous work related to it. In Section 5 we highlight our vision for solving this problem, and by Section 6 we conclude the paper.

\section{BACKGROUND}

The HEART platform underpins a software product line that allows deriving specific variants of the HEART architecture. Examples of typical variation points are the heterogeneous devices owned by users, and their technological capabilities, i.e. if they are driven by operational systems (OS) (e.g. smartphone with camera, microphone, etc.) or devices that are driven by firmware (e.g. smart watch with accelerometer, gyroscope, etc.), as well as devices that are

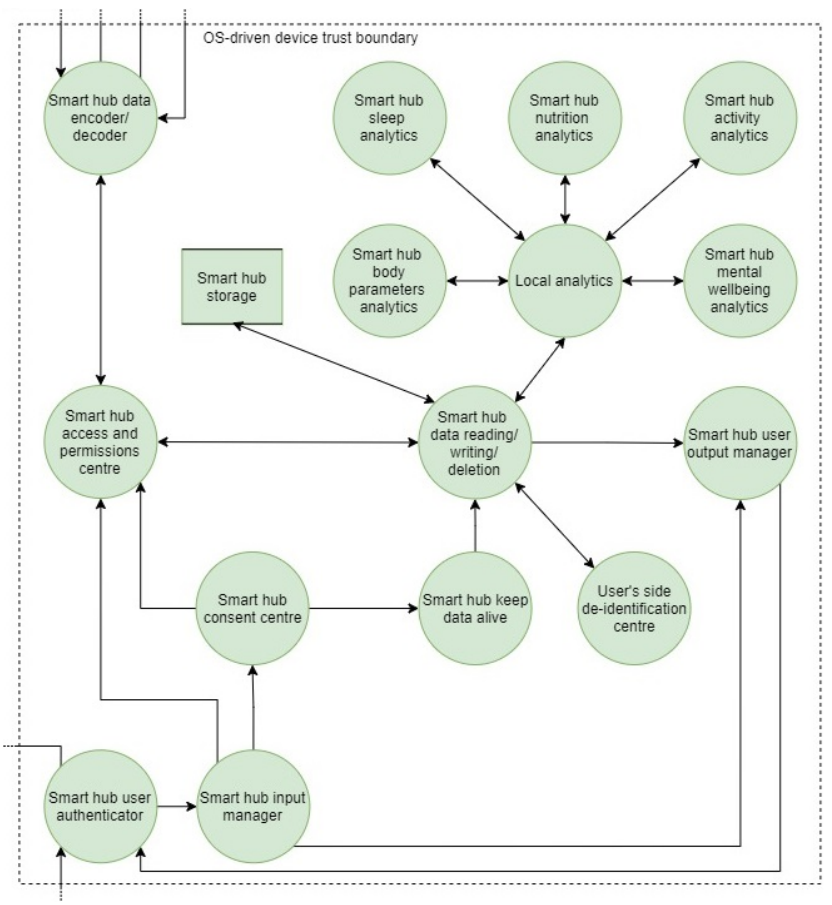

Figure 2: Part of the reference architecture DFD that represents the OS-driven device

owned by data controllers or data processors (e.g. servers with datadriven insights on environmental data, electronic health records, etc.).

Specific architectural variants derived from the HEART product line should also be tailored to specific project use cases. In our project they are healthy living, disease prevention, population health management, support of elderly, and parenting. The conceptual difference between the use cases contribute to different feature sets that are required for an architectural variant to have. For example, healthy living use case requires generic data on activity, sleep, and nutrition of the end user, while disease prevention use case may require precise information on the exercises performed or amount of specific nutrients consumed.

In order to navigate the feature space of our product line and obtain architectural variants, we have created a HEART feature model (FM) which is available at [20]. The specifics of the architectural variants derivation are out of scope for this paper. A part of the feature model that represents data processing aspect of the OS-driven device can be found on Figure 1.

For representing processes that rely on functional features and relations between them, we created a data flow diagram (DFD) of our reference architecture which can also be found at [20]. Figure 2 represents a part of this diagram. The depicted part consists of processes (e.g. User's side de-identification), data flows (e.g. line between Smart hub input manager and Smart hub access and permissions centre), and data storages (e.g. Smart hub storage) of the OS-driven device. DFDs also usually include entities (e.g. Data controller), but they are out of scope for this figure. 


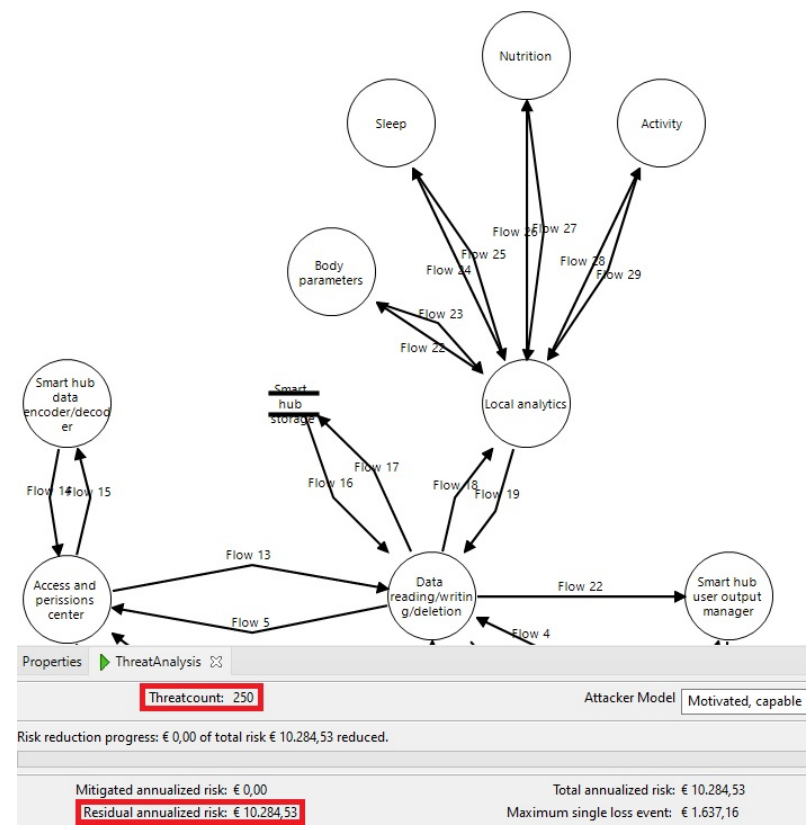

(a) Analytics of mental well-being not involved

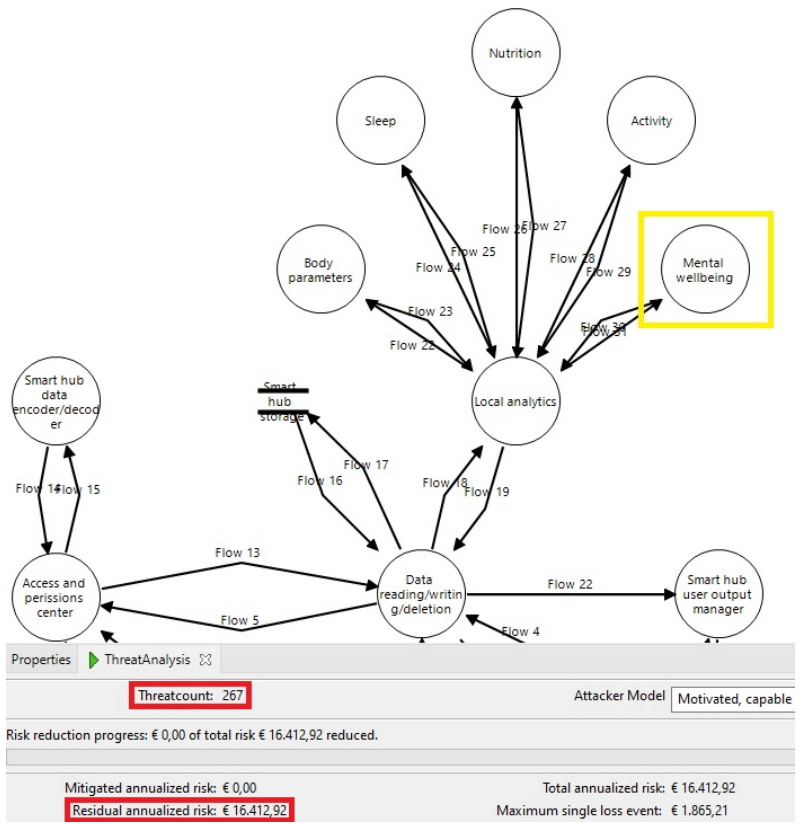

(b) Analytics of mental well-being involved

Figure 3: Results of threat and risk assessments of OS-driven device segment of two architectural variants with the usage of SPARTA tool. The difference between the architectural variants is only in presence or absence of a process responsible for analytic on "Mental well-being"

Since the current design of the platform supports analytics of sleep, activity, nutrition, body parameters, mental well-being, environment, and medical records, the specific requirements to architectural variant foreseen for a certain use case will dictate whether minimal (e.g. the usage of activity-related data only), maximal (the usage of all the available and functionally necessary data sources), or any architectural variant in between the two extremes is used. This also will end up in corresponding inclusion or exclusion of some of the features or data flow diagram components present on Figure 1 and Figure 2 respectively - e.g. architectural variant that does not perform nutrition analytics will posses neither "DP OSDD Nutrition" with its child features in its FM, nor "Smart hub nutrition analytics" in its DFD.

In terms of security and privacy analytics, for identifying which countermeasures need to be present in a given architectural variant, we decided to apply STRIDE [11] and LINDDUN [6]. These are established and accepted threat modelling approaches for security and privacy respectively [17]. Furthermore, we employed SPARTA [18] which extends the methodologies in question with FAIR [7] for the sake of risk assessment, and automates them, since manual execution of STRIDE and LINDDUN requires expert involvement and is costly as well as timeconsuming. Also, this tool is Eclipse-based, which allows its further integration in an automated derivation and assessment pipeline for different architectures.

\section{IDENTIFIED PROBLEM}

As identified in the previous section, multiple architectural variants can be obtained from our product line and every variant has unique set of features. Despite specific aspects of functional application and case-specific requirements, and independently of whether it is a minimalistic variant limited to activity analytics or a featurerich variant that includes all possible data sources and analytics, all architectural variants will involve the collection, the processing and in some cases, the systematic disclosure to third parties of sensitive and personal health-related data. In consequence, security, privacy and regulatory compliance will be highly important in the further development of a particular variant.

Considering that we have numerous architectural variants, performing threat assessment of every variant appeals promising for reaching our goal, which is providing the best possible level of security and privacy for end users. However, such an approach is not widely accepted in industry. The reason is that threat and risk assessment is time and resource-consuming activity, and in case of large architectures, a thorough assessment may take days or even weeks for an expert to complete.

In our case, we started with using SPARTA for performing threat modelling and risk assessment for a part of our product line depicted on Figure 2. This tool provided us with automated threat assessment with the usage of STRIDE, LINDDUN and FAIR methodologies, and brought calculations of risks as an output. More insights on how the methodologies that are employed by SPARTA operate is available in [17], [21], and [2] respectively.

After a successful completion of the assessment for an OS-driven device segment of a variant (results depicted on Figure 3 (a)), we performed the same manipulations with an architectural variant which 
is highly similar and differentiates only by one process (results depicted on Figure 3 (b)). Subsequently, the results of the assessment of both architectural variants were significantly different in terms of the number of threats and residual risk, which is also depicted on Figure 3.

It is unsurprising that there can be a significant difference in terms of security and privacy when a system processes a limited amount of data types with limited precision and quantity (e.g. accelerometer data in the context of activity analytics) in comparison to a more complete variant that processes the full variety of data types that the HEART platform supports, but such behaviour was not expected in case of minor differences in terms of architecture.

Performing a closer analysis of the architectural variants enabled us to locate sources of increased risk. Despite that variant $b$ has slightly larger threat surface than variant $a$ due to an extra process and a couple of data flows, as it can be seen in Figure 1 it also involves data from a device's microphone into the processing. Furthermore, "Mental wellbeing" process uses camera not for classification of objects, like it is for the case of "Nutrition" process, but for recognition of end user's facial expressions which in its turn is highly sensitive from the privacy point of view. Extrapolation of such finding leads to the fact that in our architectural variants security and privacy threats and risks are more impacted by the concrete application and the use case and less by the architectural structure itself. Moreover, the role of non-functional requirements in our situation also should not be underestimated here, but investigation on such aspect is out of scope for this paper.

Summarizing our experience with regards to architectural divergence, it can be seen that the problem lays in a fact that a highly similar architectural variants in terms of the functionality, give rise to drastically different threat and risk landscapes. This exacerbates even further when we take into account product lines like the HEART one, which has many architectural variants, and almost every variant has many threats. Furthermore, in their turn, threats can be mitigated in various ways, and complication becomes even greater when regulatory as well as user requirements also have to be met.

\section{EXISTING APPROACHES TO THE ASPECTS OF THE HIGHLIGHTED PROBLEM}

If we disassemble the problem to find a way of solving it, we end up in several aspects efficient addressing of which may lead to reaching our previously highlighted goal. Such aspects include (i) effective modelling of architectural variants, (ii) efficient threat assessment, (iii) a recommendation engine for architectural variants, and a (iv) requirements checker. This section provides a brief overview of the current state-of-the-art on the identified aspects.

Architectural variability. Modelling of architectural variants is a relatively well-researched topic, but in identified relevant papers security and privacy aspects are not well covered. For example, in [8] the authors present an approach that provides a unified perspective to users configuring products, which is aimed at improving variability modelling. Nevertheless, security and privacy aspects of architectural variability remained ignored by this paper. In [4] authors tried to approach variability modelling and proposed a framework for assessing the expressiveness of a process modelling languages, but their perspective was focused on business processes, and thus security aspects of variability in various solutions were not covered. In [19] authors introduced an approach for identifying and visualizing variability within object-oriented code, but matching their perspective with threat assessment in the context of architectural variability is far from straightforward.

Systematic threat assessment of product line variants. When we look at the threat assessment aspect within our context, there are several contributions of interest. In [12] authors propose a dynamic risk management system that collects data from multiple sources, detects anomalies, models attacks, and even automatically infers the best response actions. However, value of such contribution for our case is limited because it focuses on organizational assets and does not cover architectural variability aspects. In [3] authors introduce a methodology in support of the security analysis of an IoT system, but their solution has several limitations: $(i)$ it requires major human intervention at the threat modelling as well as risk analysis steps which makes it costly to systematically compare different variants, (ii) the proposed threat modelling approach is rather high level, (iii) risk analysis is based on a simple model that relies on likelihood and impact the assessment of which is largely dependent on human/expert input. In contrast to [3], authors of [18] provide a more exhaustive approach to threat assessment. Furthermore, their tooling connects threats and risks with potential business losses that are more actionable for the business. Furthermore, in the latter paper authors employ FAIR for risk assessment which leads to more reproducible as well as more justified assessments. However, both papers do not take into account architectural variability and its influence on the threat landscape of the system as well as its potential configurations.

Configuration support. Coming to the aspect of configuration recommendations, existing literature again has gaps. For example, in [10] authors presented a process to help developers to automatically generate suitable configurations of functional attributes. Nonetheless, the paper focused mainly on efficiency and performance. In [5] authors present a solution to assist industrial practitioners in producing a safe and secure solution out of numerous architectural variants but their solution is based on certain architectural patterns and synthesis of model-based architectural variants which does not cover the full scope of potential architectural variants and also leaves space for vulnerabilities. Taking a "user first" approach, authors of [15] proposed a feature recommender system that provides recommendations on system configurations. However, security and privacy by design as well as compliance of a system with regulations is assured by developers and architects, thus proposed solution has limited value and another one that relies on threat scope and satisfaction of requirements should be in place. Considering developers' perspective, in [14] authors propose a methodology for ensuring security in a software product line that allows to annotate the system design model with productline variability and security requirements. This work also offers a family-based security analysis within the methodology that relies on a pre-selected UMLsec checks. However, such an approach significantly depends on the the expert knowledge which is often not available. Furthermore, more prominent role of the expert makes the results of the analysis less reproducible. 
Constraint satisfaction. In terms of the aspect of meeting requirements, in [13] authors proposed an approach that contributes to satisfaction of stakeholders' requirements and feature models' constraints. In their work security is considered as a kind of nonfunctional requirements, which is not always the case. Furthermore, the authors make conclusions in their solutions with regards to security without employing recognised and/or standardised security threat modelling techniques.

\section{VISION AND ONGOING WORK}

We first deduce the a priori motivation for addressing the highlighted problem in the context of security and privacy oriented product lines. Subsequently, we sketch the envisioned approach to address the problem in the context of our ongoing work related to the eHealth IoT platform and corresponding product line.

Overall problem motivation. The highlighted problem is a potential impediment in approaches that provide architectural design support in an exhaustive, systematic manner by generating a number of variants of architectures and assessing these. If the security and privacy variability that emerges from this problem cannot be captured or characterized, the mitigation of these risks in the design of a product line -for example at the basis of a reference architecture as discussed above- will necessarily remain a manual and costly activity for each individual application case. This in turn significantly reduces the effectiveness of an overall product line approach that is rooted upon systematically capturing the sources of variation and diversity among candidate architectures.

Envisioned approach. Considering the existing state of-the-art, we see a clear need for a solution that delivers a recommendation on architectural configurations for system/security architects and developers. Such a recommendation should explicitly model and take into account the architectural variability, outcomes of threat and risk assessment for every architectural variant, and overall compliance of every system configuration with relevant requirements as well as regulations. We envision that such a solution may rely on existing efforts, with extensions and integrations that are necessary for solving the identified problem.

In our opinion, the most prominent opportunity for obtaining a solution of interest is extension of the tooling introduced in [18], as it employs established and accepted methodologies [17]. Establishing a tool chain that employs it in combination with other necessary components can enable creation of a solution for the problem that we highlighted. Accordingly to the product line engineering framework depicted in [16], we envision such a toolchain as depicted in Figure 4.

\section{CONCLUSION}

This paper discusses and demonstrates a specific problem encountered in the establishment of a software product line that addresses the functional variability of an eHealth IoT platform which supports a wide range of health-related use cases.

In its essence, the problem is that two architectural variants supported by the product line, despite them being highly similar in terms of functionality and supporting architectural structures, give rise to fundamentally different threat landscapes. In consequence,

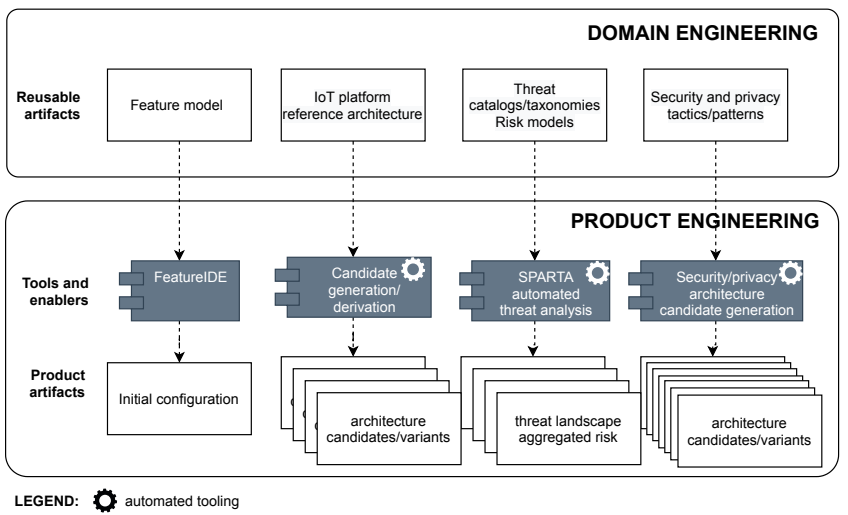

Figure 4: The envisioned tool chain for addressing security variability in a an automated search-based approach

a degree of architectural variability that is more difficult to capture and manage is introduced: the different ways to address these threats, the architectural security and privacy countermeasures and mitigations.

We have illustrated this problem with an example involving two variant architectures which we have subjected to automated threat analysis (at the basis of STRIDE and LINDDUN threat modeling approaches) and exhaustive risk assessment. In addition, we foresee and discuss a solution for such a problem in the form of an automated pipeline that conducts a targeted search activity through the space of architecture variants, submits them to automated threat analysis and then investigates a consecutive search activity specifically in the space of the architectural security and privacy mitigations (e.g. at the basis of security tactics or patterns). The outcome could then be a set of candidate architectures, combined with guidance and evaluation results that indicate the extent to which these candidates will satisfy the different requirements, and which can serve as a basis for architectural trade-off decision making.

The characterization of this problem as presented in this paper is a first stepping stone in our ongoing work towards the complete establishment of the described product line that takes place in the HEART project [1]. These efforts are more specifically aimed at the longer-term research goal to show and evaluate the effectiveness and feasibility of approaches that implement a thorough, toolsupported search to generate and automatically evaluate different architectural candidates, in line with the principles of search-based software engineering [9].

\section{ACKNOWLEDGMENTS}

This research is funded by Philips Research, Research Fund KU Leuven, and the HEART project (www.heart-itn.eu). This project has received funding from the European Union's Horizon 2020 research and innovation programme under the Marie SkłodowskaCurie grant agreement No 766139. This publication reflects only the authors' view and the REA is not responsible for any use that may be made of the information it contains. 


\section{REFERENCES}

[1] [n. d.]. HEalth related Activity Recognition system based on IoT. http://heart-itn $\mathrm{eu} /$

[2] 2015. Measuring and managing information risk: a FAIR approach (1st edition ed.). Butterworth-Heinemann., Burlington.

[3] 2019. Toward the automation of threat modeling and risk assessment in IoT systems. Internet of Things 7 (2019), 100056. https://doi.org/10.1016/j.iot.2019. 100056

[4] Clara Ayora, Victoria Torres, Barbara Weber, Manfred Reichert, and Vicente Pelechano. 2015. VIVACE: A framework for the systematic evaluation of variability support in process-aware information systems. Information and Software Technology 57 (2015), 248-276. https://doi.org/10.1016/j.infsof.2014.05.009

[5] Thomas Degueule, Joao Bosco Ferreira Filho, Olivier Barais, Mathieu Acher Jérôme Le Noir, Sébastien Madelénat, Grégory Gailliard, Godefroy Burlot, and Olivier Constant. 2015. Tooling Support for Variability and Architectural Patterns in Systems Engineering (SPLC '15). Association for Computing Machinery, New York, NY, USA, 361-364. https://doi.org/10.1145/2791060.2791097

[6] Mina Deng, Kim Wuyts, Riccardo Scandariato, Bart Preneel, and Wouter Joosen. 2011. A privacy threat analysis framework: supporting the elicitation and fulfillment of privacy requirements. Requirements Engineering fournal 16 (2011), $3-32$.

[7] Jack Freund and Jack Jones. 2014. Measuring and Managing Information Risk: A FAIR Approach. Butterworth-Heinemann, USA.

[8] José A. Galindo, Deepak Dhungana, Rick Rabiser, David Benavides, Goetz Botterweck, and Paul Grünbacher. 2015. Supporting distributed product configuration by integrating heterogeneous variability modeling approaches. Information and Software Technology 62 (2015), 78-100. https://doi.org/10.1016/j.infsof.2015.02. 002

[9] Mark Harman, S Afshin Mansouri, and Yuanyuan Zhang. 2012. Search-based software engineering: Trends, techniques and applications. ACM Computing Surveys (CSUR) 45, 1 (2012), 1-61.

[10] Jose-Miguel Horcas, Mónica Pinto, and Lidia Fuentes. 2018. Variability models for generating efficient configurations of functional quality attributes. Information and Software Technology 95 (2018), 147-164. https://doi.org/10.1016/j.infsof.2017. 10.018

[11] Loren Kohnfelder and Praerit Garg. 1999. The threats to our products. Microsoft Interface (April 1999)
[12] Xavier Larriva-Novo, Mario Vega-Barbas, Victor A. Villagrá, Diego Rivera, Mario Sanz, and Manuel Âlvarez Campana. 2020. Dynamic Risk Management Architecture Based on Heterogeneous Data Sources for Enhancing the Cyber Situational Awareness in Organizations. In Proceedings of the 15th International Conference on Availability, Reliability and Security (ARES '20). Association for Computing Machinery, New York, NY, USA, Article 100, 9 pages. https://doi.org/10.1145/3407023.3409224

[13] Mahdi Noorian, Ebrahim Bagheri, and Weichang Du. 2017. Toward automated quality-centric product line configuration using intentional variability. fournal of Software: Evolution and Process 29, 9 (2017), e1870. https://doi.org/10.1002/ smr.1870 arXiv:https://onlinelibrary.wiley.com/doi/pdf/10.1002/smr.1870 e1870 smr.1870.

[14] Sven Peldszus, Daniel Strüber, and Jan Jürjens. 2018. Model-Based Security Analysis of Feature-Oriented Software Product Lines. 53, 9 (Nov. 2018), 93-106. https://doi.org/10.1145/3393934.3278126

[15] Juliana Alves Pereira, Pawel Matuszyk, Sebastian Krieter, Myra Spiliopoulou, and Gunter Saake. 2016. A Feature-Based Personalized Recommender System for Product-Line Configuration. SIGPLAN Not. 52, 3 (Oct. 2016), 120-131. https: //doi.org/10.1145/3093335.2993249

[16] Klaus Pohl, Günter Böckle, Frank van der Linden, and Gunter Bc6ckle. 2005. Software Product Line Engineering: Foundations, Principles, and Techniques. Springer Berlin / Heidelberg, Berlin, Heidelberg.

[17] Adam Shostack. 2014. Threat Modeling: Designing for Security (1st ed.). Wiley Publishing.

[18] Laurens Sion, Dimitri Van Landuyt, Koen Yskout, and Wouter Joosen. 2018. SPARTA: Security Privacy Architecture Through Risk-Driven Threat Assessment. In 2018 IEEE International Conference on Software Architecture Companion (ICSAC). 89-92. https://doi.org/10.1109/ICSA-C.2018.00032

[19] Xhevahire Tërnava, Johann Mortara, and Philippe Collet. 2019. Identifying and Visualizing Variability in Object-Oriented Variability-Rich Systems (SPLC '19). Association for Computing Machinery, New York, NY, USA, 231-243. https: //doi.org/10.1145/3336294.3336311

[20] Oleksandr Tomashchuk. [n. d.]. Supporting materials for publication "The architectural divergence problem in security and privacy of eHealth IoT product lines". https://u.pcloud.link/publink/show?code= kZ68cMXZlVp5Xh9jB8Hetl3r3MwMXb1E4Tgy

[21] Kim Wuyts and Wouter Joosen. 2015. LINDDUN privacy threat modeling: a tutorial. Technical Report. 